EPJ Web of Conferences 21, 07002 (2012)

DOI: $10.1051 /$ epjconf/20122107002

(C) Owned by the authors, published by EDP Sciences, 2012

\title{
Extending the Kawai-Kerman-McVoy Statistical Theory of Nuclear Reactions to Intermediate Structure via Doorways
}

\author{
G. Arbanas ${ }^{1, a}$, C. A. Bertulani ${ }^{2}$, D. J. Dean ${ }^{1}$, A. K. Kerman ${ }^{3,4}$, and K. J. Roche ${ }^{5}$ \\ 1 Oak Ridge National Laboratory, P.O. Box 2008, Oak Ridge, TN 37831-6171, USA \\ 2 Texas A\&M University - Commerce, P.O. Box 3011, Commerce, TX 75429, USA \\ 3 Massachusetts Institute of Technology, 77 Massachusetts Ave., 6-306, Cambridge, MA 02139, USA \\ 4 The University of Tennessee, Knoxville, TN, USA \\ 5 Pacific Northwest National Laboratory, P.O. Box 999, Richland, Washington 99352 and The Uni- \\ versity of Washington, Box 351560, 3910 15th Ave. NE, Seattle, WA 98195-1560, USA
}

\begin{abstract}
Kawai, Kerman, and McVoy have shown that a statistical treatment of many open channels that are coupled by direct reactions leads to modifications of the HauserFeshbach expression for energy-averaged cross section [Ann. of Phys. 75, 156 (1973)]. The energy averaging interval for this cross section is on the order of the width of single particle resonances, $\approx 1 \mathrm{MeV}$, revealing only a gross structure in the cross section. When the energy-averaging interval is decreased down to a width of a doorway state, $\approx 0.1 \mathrm{MeV}$, a so-called intermediate structure may be observed in cross sections. We extend the Kawai-Kerman-McVoy theory into the intermediate structure by leveraging a theory of doorway states developed by Feshbach, Kerman, and Lemmer [Ann. of Phys. 41, 230 (1967)]. As a by-product of the extension, an alternative derivation of the central result of the Kawai-Kerman-McVoy theory is suggested. We quantify the effect of the approximations used in derivation by performing numerical computations for a large set of compound nuclear states.
\end{abstract}

\section{Introduction}

One way of analyzing low-energy nuclear cross sections is by varying the experimental energy resolution, or alternatively, by numerical energy-averaging of high-resolution data. It is known that different energy resolutions may reveal different features in the cross section. Such features may provide insight into the dominant processes contributing to the cross section.

Extremely high energy resolution, on the order of fraction of an $\mathrm{eV}$, reveals compound nuclear resonances, often referred to as fine structure. In the other extreme, when the energy resolution is on the order of MeV's, single-particle resonances may remain the only visible feature of what is commonly referred to as a gross structure [1]. For an intermediate energy resolution, on the order of $100 \mathrm{keV}$, a so-called intermediate structure emerges, for which a theory of doorway states was developed in [2]. Doorway state concept was used to construct a non-local optical potential in [6].

In this work we will consider the intermediate and the gross structures of low-energy cross sections. The latter is the realm of the optical potentials and statistical theories of nuclear reactions, of which we

a e-mail: arbanasg@ornl.gov

Notice: This manuscript has been authored by UT-Battelle, LLC, under contract DE-FC02-09ER41583 (UNEDF SciDAC Collaboration) with the U.S. Department of Energy. The United States Government retains and the publisher, by accepting the article for publication, acknowledges that the United States Government retains a nonexclusive, paid-up, irrevocable, world-wide license to publish or reproduce the published form of this manuscript, or allow others to do so, for United States Government purposes.

This is an Open Access article distributed under the terms of the Creative Commons Attribution-Noncommercial License 3.0, which permits unrestricted use, distribution, and reproduction in any noncommercial medium, provided the original work is properly cited. 


\section{EPJ Web of Conferences}

will focus on the Kawai-Kerman-McVoy (KKM) theory [1], which will be outlined in Sect. 2. We will leverage some of the formal expressions derived in [2] to extend the KKM theory into the realm of the intermediate structure in Sect. 3. We will test the validity of some approximations used in derivation of KKM results using a simple numerical model in Sect. 4.

\section{Gross Structure}

In this section we outline some key ingredients of the KKM theory [1]. Alternative expositions of the KKM theory can be found in [7] and [5]. The theory uses the Feshbach's projection operator formalism to divide the Hilbert space into continuum $(P)$ and the compound $(Q)$ subspaces:

$$
P+Q=1
$$

where $P$ and $Q$ are unitary Hermitian projection operators. Projecting the Schrodinger equation $H \Psi=$ $E \Psi$ into the two subspaces, and using a two-potential formula to find the $T$-matrix, yields a backgroundplus-resonant expression ${ }^{1}$ :

$$
T=T_{P}+T_{Q},
$$

where $T_{P}$ is the $T$-matrix of the $P H P \equiv H_{P P}$, and ${ }^{2}$

$$
T_{Q}=H_{P Q} \frac{1}{E-H_{Q Q}-W_{Q Q}} H_{Q P}
$$

where a complex symmetric operator

$$
W_{Q Q}=H_{Q P} \frac{1}{E^{(+)}-H_{P P}} H_{P Q}
$$

describes coupling of compound states to the continuum which gives compound states finite widths. Expanding the $T_{Q}$ in terms of energy-dependent eigenvectors and (complex) eigenvalues

$$
\left[H_{Q Q}+W_{Q Q}\right]|\hat{q}\rangle=\hat{E}_{q}|\hat{q}\rangle
$$

yields

$$
T_{Q, c c^{\prime}}=\frac{1}{2 \pi} \sum_{q} \frac{\hat{g}_{q c} \hat{g}_{q c^{\prime}}}{E-\hat{E}_{q}}
$$

where partial width amplitudes $\hat{g}_{q c}$ are given by

$$
\hat{g}_{q c} \equiv\left\langle c^{(-)}\left|H_{P Q}\right| \hat{q}\right\rangle
$$

where $\left|c^{(-)}\right\rangle$is an eigenfunction of $H_{P P}$ in channel $c$ with incoming boundary condition. A disadvantage of the background-plus-resonant separation in Eq. (2) is that computation of its energy-averaged cross section will contain a non-vanishing energy-average of the product $T_{P} T_{Q}$. The KKM theory solves this problem by deriving an alternative separation of the $T$-matrix into its optical (i.e., energy-averaged) and fluctuating parts:

$$
T \approx T^{\mathrm{opt}}+T^{\mathrm{fluct}}
$$

where $T^{\mathrm{opt}}$ is the $T$-matrix of the optical Hamiltonian $H^{\mathrm{opt}}$ and

$$
H^{\mathrm{opt}}=H_{P P}+H_{P Q} \frac{1}{E-H_{Q Q}+i I / 2} H_{Q P},
$$

\footnotetext{
${ }^{1}$ To simplify the notation, energy dependence of the $T$-matrix will not be displayed in this paper.

${ }^{2}$ In a conventional notation subscripts refer to subspace projection operators: $H_{P Q} \equiv P H Q, W_{Q Q} \equiv Q W Q$, etc.
} 


\section{$\mathrm{CNR} * 11$}

where $I$ is the energy-averaging interval that is on the order of $1 \mathrm{MeV}$ for optical potentials. The KKM expression for $T^{\text {fluct }}$ is:

$$
T^{\text {fluct }}=V_{P Q} \frac{1}{E-H_{Q Q}-W_{Q Q}^{\text {opt }}} V_{Q P},
$$

where $W_{Q Q}^{\mathrm{opt}}$ is obtained by substituting $H_{P P} \rightarrow H_{P P}^{\mathrm{opt}}$ and $H_{P Q} \rightarrow V_{P Q}$ in Eq. (4), and where

$$
V_{P Q} \equiv H_{P Q} \sqrt{\frac{i I / 2}{E-H_{Q Q}+i I / 2}} .
$$

Expanding the $T^{\text {fluct }}$ in terms of energy-dependent eigenvectors and (complex) eigenvalues,

$$
\left[H_{Q Q}+W_{Q Q}^{\mathrm{opt}}\right]|q\rangle=E_{q}|q\rangle
$$

yields

$$
T_{c c^{\prime}}^{\text {fluct }}=\frac{1}{2 \pi} \sum_{q} \frac{g_{q c} g_{q c^{\prime}}}{E-E_{q}}
$$

where partial width amplitudes $g_{q c}$ are given by

$$
g_{q c} \equiv\left\langle c_{\mathrm{opt}}^{(-)}\left|V_{P Q}\right| q\right\rangle
$$

and where $\left|c_{\mathrm{opt}}^{(-)}\right\rangle$is an optical wave-function in channel $c$ with incoming boundary condition. The advantage of this expression is that the energy-average of the fluctuating term is approximately zero because by construction the optical $T^{\mathrm{opt}}$ is the energy-average of the $T$-matrix:

$$
T^{\mathrm{opt}} \approx\langle T\rangle_{I} .
$$

Computation of energy-averaged cross section, being proportional to $|T|^{2}$, is therefore simplified because the energy-average of the cross term is negligible. Using this feature, the KKM derived an expression for the energy-averaged cross section:

$$
\left\langle\sigma_{c c^{\prime}}^{\text {fluct }}\right\rangle_{I} \approx(2 l+1) \pi \lambda_{c}^{2} \frac{P_{c c} P_{c^{\prime} c^{\prime}}+P_{c c^{\prime}} P_{c^{\prime} c}}{\operatorname{Tr}(P)},
$$

where the Satchler's penetrability matrix $P$ is computed for $H^{\text {opt }}$. The same feature was also used in derivation of the Kerman-McVoy energy-averaged cross section for two-step (direct-plus-compound) reactions [3], and also in derivation of the Feshbach-Kerman-McVoy statistical theory of multistep pre-equilibrium reactions [4].

\section{Intermediate Structure}

We set out to express the $T$-matrix of intermediate structure as a sum of its average and fluctuating parts, analogous to Eq. (8) of the gross structure. We leverage the separation of the $T$-matrix into its background-plus-resonant parts, which was derived using Feshbach projection operators formalism in [2]. The Hilbert space is projected into continuum $(P)$, doorway $(D)$, and compound $(F)$ subspaces:

$$
P+D+F=1,
$$

where $D+F=Q$ of Sect. 2. Projecting the Schrodinger equation $H \Psi=E \Psi$ into these three subspaces, and allowing compound subspace to couple to doorways only $\left(P H D \equiv H_{P D} \neq 0, H_{D F} \neq 0\right.$, and $H_{P F}=0$ ), it was shown in [2] that a $T$-matrix can be formally written as

$$
T=T_{P}+T_{D}+T_{F},
$$




\section{EPJ Web of Conferences}

which is the intermediate structure analogue of the background-plus-resonant $T$-matrix in Eq. (2). Of the three terms, the most rapid energy dependence occurs in $T_{F}$ because it contains the effect of the compound resonant states. For completeness we copy the expressions for $T_{D}$ and $T_{F}$ from Eq. (2.40) of [2]:

$$
\begin{aligned}
& T_{D}=H_{P D} \frac{1}{E-H_{D D}-W_{D D}} H_{D P} \\
& T_{F}=H_{P D} \frac{1}{E-H_{D D}-W_{D D}} H_{D F} \frac{1}{E-H_{F F}-H_{F D} \frac{1}{E-H_{D D}-W_{D D}} H_{D F}} H_{D F} \frac{1}{E-H_{D D}-W_{D D}} H_{D P},
\end{aligned}
$$

where

$$
W_{D D}=H_{D P} \frac{1}{E^{(+)}-H_{P P}} H_{P D} .
$$

The identities used in the KKM to cast the $T$-matrix into a sum of its average and fluctuating parts in Eq. (8) cannot be used when doorway space $D$ is treated explicitly. A desired separation of the $T$-matrix into its average and fluctuating parts is accomplished below by expressing the $T$-matrix completely in terms of scalar matrix elements. Despite this departure from the KKM, the approximations used in the derivation below are consistent with those of the KKM, so that the results below ought to be consistent with those of the KKM. To proceed, we expand the $T_{F}$ in terms of eigenvectors ${ }^{3}$ and (complex) eigenvalues of the operator expressions appearing in $T_{F}$ :

$$
\begin{aligned}
\left(H_{D D}+W_{D D}\right)|d\rangle & =E_{d}|d\rangle \\
\left(H_{F F}+H_{F D} \frac{1}{E-H_{D D}-W_{D D}} H_{D F}\right)|\lambda\rangle & =E_{\lambda}|\lambda\rangle .
\end{aligned}
$$

The expansion yields

$$
T_{F, c c^{\prime}}=\frac{1}{2 \pi} \sum_{\lambda} \frac{G_{\lambda c} G_{\lambda c^{\prime}}}{E-E_{\lambda}}
$$

where the composite partial widths $G_{c \lambda}$,

$$
G_{\lambda c}=\sum_{d} \frac{\gamma_{c d} \gamma_{d \lambda}}{E-E_{d}}
$$

are defined in terms of partial widths

$$
\begin{aligned}
\gamma_{c d} & =\left\langle c^{(-)}\left|H_{P D}\right| d\right\rangle \\
\gamma_{d \lambda} & =\left\langle\tilde{d}\left|H_{D F}\right| \lambda\right\rangle,
\end{aligned}
$$

where $\langle\tilde{d}|$ is a bi-orthogonal counterpart of $|d\rangle$. Next, we energy-average the $T_{F, c c^{\prime}}$ in Eq. (24) over an intermediate energy-averaging interval, $I_{\text {int }}$, that is smaller than a doorway state width $\Gamma_{d}$ (in order to preserve intermediate structure) but is much larger than the width of fine compound resonances $\Gamma_{\lambda}$ (in order to smooth out the fine structure), or

$$
\Gamma_{\lambda} \ll I_{\text {int }}<\Gamma_{d} \ll I
$$

Since $T_{P}$ and $T_{D}$ in Eq. (18) are assumed not to vary appreciably over the energy-averaging interval $I_{\text {int }}$, the effect of this energy-averaging on these terms is neglected. Similarly, the effect of energy-averaging the composite partial widths $G_{c \lambda}$ over the same energy-averaging interval $I_{\text {int }}$ is assumed to be small. Therefore, only the narrow compound resonance poles, $E_{\lambda}$, of Eq. (24) need to be energy-averaged.

\footnotetext{
3 These eigenvectors and their corresponding (complex) eigenvalues are energy-dependent because the operator expressions are explicitly energy-dependent. The imaginary parts of the complex eigenvalues $\operatorname{Im}\left(E_{d}\right)=\Gamma_{d} / 2$ and $\operatorname{Im}\left(E_{\lambda}\right)=\Gamma_{\lambda} / 2$ give the resonant widths of doorway and compound states, respectively.
} 


\section{$\mathrm{CNR} * 11$}

Energy-averaging with a Lorentzian weight of width $I_{\text {int }}$ can be performed by contour integration ${ }^{4}$ that, for a function $T(E)$ that is regular in the upper half-plane, yields

$$
\langle T(E)\rangle_{I_{\text {int }}}=T\left(E+i I_{\text {int }} / 2\right) .
$$

Application of this analytical result to Eq. (24), along with the approximations just described, yields

$$
\left\langle T_{F, c c^{\prime}}\right\rangle_{I_{\text {int }}} \approx \frac{1}{2 \pi} \sum_{\lambda} \frac{G_{c \lambda} G_{\lambda c^{\prime}}}{E-E_{\lambda}+i I_{\text {int }} / 2} .
$$

An expression for the fluctuating part of the $T$-matrix can now be determined from its definition:

$$
\begin{aligned}
T_{F}^{\text {fluct }} & \equiv T-\langle T\rangle_{I_{\text {int }}} \\
& \approx T_{F}-\left\langle T_{F}\right\rangle_{I_{\text {int }} .} .
\end{aligned}
$$

Inserting Eqs. (24) and (30) into Eq. (32), and displaying the channel indices, yields

$$
T_{F, c c^{\prime}}^{\text {fluct }} \approx \frac{1}{2 \pi} \sum_{\lambda} \frac{\mathcal{G}_{\lambda c} \mathcal{G}_{\lambda c^{\prime}}}{E-E_{\lambda}},
$$

where

$$
\mathcal{G}_{\lambda c}=G_{\lambda c} \sqrt{\frac{i I_{\text {int }} / 2}{E-E_{\lambda}+i I_{\text {int }} / 2}} .
$$

Now that we derived an expression for the fluctuating part, $T_{F}^{\text {fluct }}$, we turn our attention to the intermediate energy-averaged $T$-matrix. For the purposes of this work, it suffices to state a formal expression for the intermediate structure Hamiltonian, given in Eq (2.89) of [2], which for energy-averaging with a Lorentzian weight of width $I_{\text {int }}$, becomes

$$
H^{\mathrm{int}}=H_{P P}+H_{P D} \frac{1}{E-H_{D D}-H_{D F} \frac{1}{E-H_{F F}+i_{\text {int }} / 2} H_{F D}} H_{D P} .
$$

Since this $H^{\text {int }}$ is constructed so that its $T$-matrix is approximately equal to the intermediate energyaverage of the total $T$-matrix, namely,

$$
T^{\mathrm{int}} \approx\langle T\rangle_{\text {int }},
$$

we arrive at a desired separation of the $T$-matrix:

$$
T \approx T^{\text {int }}+T_{F}^{\text {fluct }} .
$$

This separation of the $T$-matrix into its intermediate energy-average plus a fluctuating part is analogous to the KKM separation into optical plus its fluctuating part. It is this separation that makes it possible to retrace the steps in the derivation of the KKM cross section. Doing so yields an expression for the intermediate-structure cross section arising from intermediate energy-averaging of $\left|T_{F}^{\text {fluct }}\right|^{2}$ in Eq. (33):

$$
\left\langle\sigma_{F, c c^{\prime}}^{\text {fluct }}\right\rangle_{\text {int }} \approx(2 l+1) \pi \lambda_{c}^{2} \frac{P_{c c} P_{c^{\prime} c^{\prime}}+P_{c c^{\prime}} P_{c^{\prime} c}}{\operatorname{Tr}(P)} .
$$

This expression is identical in form to the KKM expression in Eq. (16), the only difference being that Satchler's penetrability matrix $P$ is computed for $H^{\text {int }}$ instead of $H^{\text {opt }}$.

\footnotetext{
${ }^{4}$ See for example Eq. (2.19) of [2] for more details.
} 


\subsection{KKM Revisited}

The derivation of the fluctuating $T$-matrix for the intermediate structure in Sect. 3 could be used to derive an alternative to Eq. (13). Writing the fluctuating $T$-matrix as in Eq. (31) but using the energyaveraging interval of the gross structure, $I$, yields

$$
\begin{aligned}
T_{Q}^{\text {fluct }} & \equiv T-\langle T\rangle_{I} \\
& \approx T_{Q}-\left\langle T_{Q}\right\rangle_{I} .
\end{aligned}
$$

Energy-averaging of $T_{c c^{\prime}}^{Q}$ in Eq. (6) using a Lorentzian weight of width $I$, and performing the subtraction above, yields

$$
T_{Q, c c^{\prime}}^{\mathrm{fluct}}=\frac{1}{2 \pi} \sum_{q} \frac{\bar{g}_{q c} \bar{g}_{q c^{\prime}}}{E-\hat{E}_{q}},
$$

where

$$
\bar{g}_{c q}=\hat{g}_{c q} \sqrt{\frac{i I / 2}{E-\hat{E}_{q}+i I / 2}} .
$$

This expression is simpler than the one in Eq. (13) because it bypasses computation of eigenvectors $|q\rangle$ and eigenvalues $E_{q}$ in Eq. (12). The expression for $T_{Q}^{\text {fluct }}$ above is very similar to that in Eq. (23) of [1] where it was used to argue that its energy average ought to vanish. This suggests that the expression for $T_{Q}^{\text {fluct }}$ obtained in a simplified derivation could be used to derive the KKM expression for cross section in Eq. (16).

\section{Model and Results}

A computer model for studying the validity of approximations used in the KKM derivation was constructed in [5] in order to verify numerically that $\left\langle T_{c c^{\prime}}^{\mathrm{fluct}}\right\rangle_{I} / T_{c c^{\prime}}^{\mathrm{opt}} \ll 1$. Eigenvalues and eigenvectors in Eqs. (5) and (12) were assumed not to vary appreciably over the energy-averaging range in computation of $\left\langle T_{c c^{\prime}}^{\text {fluct }}\right\rangle_{I}$. We remove this assumption in order to quantify the effect of the energy dependence of eigenvectors and eigenvalues over the energy-averaging interval.

Input parameters for this computation were otherwise mostly identical to those in [5]. $T_{c c^{\prime}}^{\text {fluct }}$ was energy-averaged with a Lorentzian weight of half-width $0.5 \mathrm{MeV}$ at an incoming energy $E=20 \mathrm{MeV}$. The Lorentzian energy average was performed over 100 equidistant points between 18 and $22 \mathrm{MeV}$. The eigenvalue Eqs. (5) and (12) were solved at each of 100 energy points spanning the energyaveraging region.

Forty s-wave channels and 1,600 compound levels were used in this computation. The random interaction was defined on $N_{R}=20$ equidistant radial points between the origin and $7 \mathrm{fm}$. The variance of the coupling strength was set to $0.5 \mathrm{MeV} \mathrm{fm}^{3 / 2}$. For simplicity, we set $H_{P P}$ to be kinetic energy, so that $T_{P}=0$, and therefore $T_{c c^{\prime}}^{\text {opt }}$ could be computed as $T_{c c^{\prime}}^{\text {opt }} \approx\left\langle T_{Q, c c^{\prime}}\right\rangle_{I}$.

A histogram of $\left\langle T_{c c^{\prime}}^{\text {fluct }}\right\rangle_{I} / T_{c c^{\prime}}^{\text {opt }}$ for $40 \times 40$ channel pairs, with and without using energy dependent eigenvectors and eigenvalues, is shown in Figure 1. The average values and square-root variances of the two histograms are displayed in Table 1. A closeness of the two results suggests that energy dependence of eigenvectors and eigenvalues may be neglected without a significant loss in accuracy.

\section{Acknowledgments}

This work was supported by the U.S. Department of Energy (DOE) under Contract No. DE-FC0209ER41583 (UNEDF SciDAC Collaboration). 
$\mathrm{CNR} * 11$

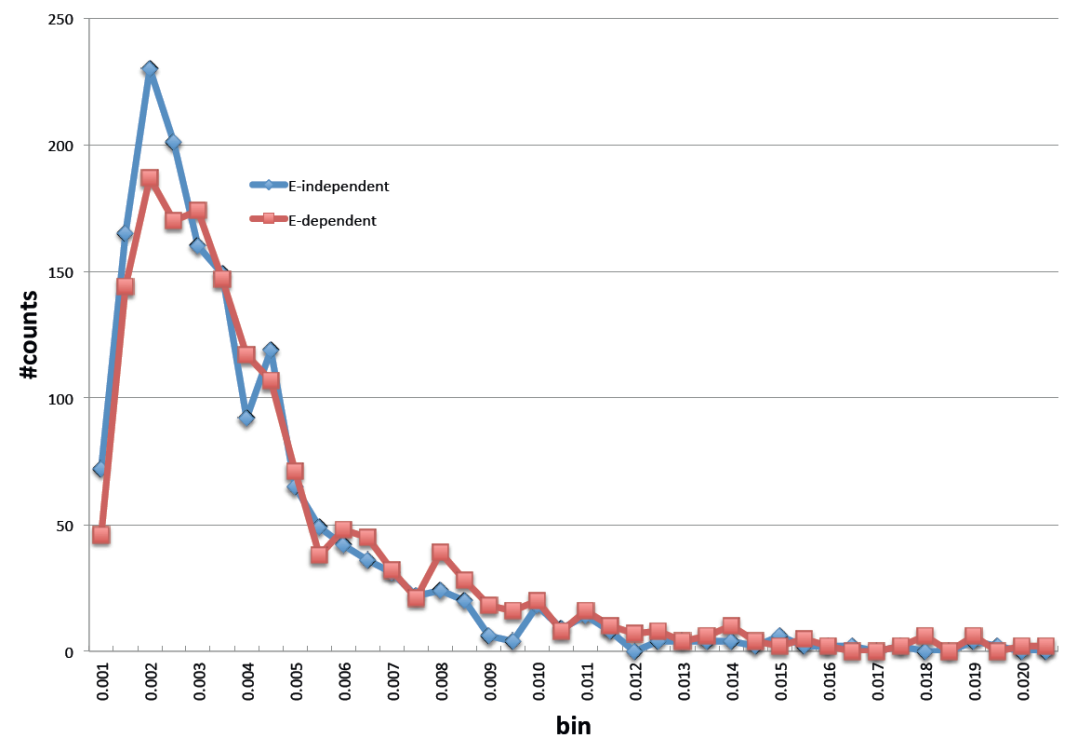

Fig. 1. A histogram of ratios $\left\langle T_{c c^{\prime}}^{\text {fluct }}(E)\right\rangle_{I} / T_{c c^{\prime}}^{\text {opt }}(E)$, computed with and without accounting for energy dependence of eigenvalues and eigenvectors. Both histograms corroborate a central result of the KKM theory $\left\langle T_{c c^{\prime}}^{\text {fluct }}(E)\right\rangle_{I} / T_{c c^{\prime}}^{\mathrm{opt}}(E) \ll 1$. A variance between the histograms is an estimate of the error caused by neglecting the energy-dependence.

Table 1. The average value and the square-root-variance for the two histograms plotted in Fig. 1. We observe a slightly larger average of the histogram for $E$-dependent eigenvectors and eigenvalues.

\begin{tabular}{lll}
\hline & Average Ratio & SQRT(Variance) \\
\hline E-independent & 0.0037 & 0.0053 \\
E-dependent & 0.0042 & 0.0049 \\
\hline
\end{tabular}

\section{References}

1. M. Kawai, A.K. Kerman, K.W. McVoy, Ann. of Phys. 75, 156-170 (1973)

2. H. Feshbach, A.K. Kerman, R.H. Lemmer, Ann. of Phys. 41, 230-286 (1967)

3. A.K. Kerman, K.W. McVoy, Ann. of Phys. 122, 197-216 (1979)

4. H. Feshbach, A.K. Kerman, S. Koonin, Ann. of Phys. 125, 429-476 (1980)

5. G. Arbanas, C.A. Bertulani, D.J. Dean, A.K. Kerman AIP Conf. Proc. 1005, 160 (2008)

6. G.P.A. Nobre, et al., Phys. Rev. Lett. 105, 202502 (2010)

7. H. Feshbach, Theoretical Nuclear Physics: Nuclear Reactions, (John Wiley \& Sons, NY, 1992) 